\title{
Schlafen-11 (SLFN11): a step forward towards personalized medicine in small-cell lung cancer?
}

\author{
Alessandro Inno ${ }^{1}$, Anna Stagno ${ }^{1,2}$, Stefania Gori ${ }^{1}$ \\ ${ }^{1}$ Medical Oncology, IRCCS Ospedale Sacro Cuore Don Calabria, Negrar, Verona, Italy; ${ }^{2}$ Medical Oncology Section, Department of Surgical, \\ Oncological and Oral Sciences, University of Palermo, Palermo, Italy \\ Correspondence to: Alessandro Inno, MD, PhD. Medical Oncology, IRCCS Ospedale Sacro Cuore Don Calabria, Via don A. Sempreboni 5, 37024 \\ Negrar, Verona, Italy. Email: alessandro.inno@sacrocuore.it. \\ Comment on: Pietanza MC, Waqar SN, Krug LM, et al. Randomized, Double-Blind, Phase II Study of Temozolomide in Combination With Either \\ Veliparib or Placebo in Patients With Relapsed-Sensitive or Refractory Small-Cell Lung Cancer. J Clin Oncol 2018;36:2386-94.
}

Submitted Nov 20, 2018. Accepted for publication Nov 28, 2018.

doi: $10.21037 /$ tlcr.2018.11.06

View this article at: http://dx.doi.org/10.21037/tlcr.2018.11.06

\section{Background}

Small-cell lung cancer (SCLC) is a highly aggressive disease with dismal prognosis (1). Given its tendency to early develop widespread metastases, in approximatively two-thirds of cases SCLC is diagnosed at extensive-stage (ES). Standard treatment for ES-SCLC has remained unchanged for years. In the first-line setting, 4-6 cycles of chemotherapy with a platinum-based drug (either cisplatin or carboplatin) plus etoposide has represented the treatment of choice for three decades $(2,3)$ whereas the combination of a platinum-based drug plus irinotecan has been considered an acceptable option, widely used in Japan (4). Despite high response rate of about $60-70 \%$ with first-line platinumbased chemotherapy, however, most patients inevitably experience progressive disease within 6 months, and median overall survival (OS) does not exceed 10 months (2-4). For patients with relapsed disease, topotecan is recognized as a standard treatment. However, topotecan achieves modest response rates of $17 \%$ in platinum-sensitive and only $5 \%$ in platinum-refractory disease, with median OS of about 3-4 months (5). Other treatments such as irinotecan, temozolomide and anthracycline-based regimens have also shown similar activity to topotecan in the second-line setting (1).

Recently, new insights into the biology of SCLC have revealed novel potential therapeutic targets including immune checkpoints, developmental regulatory pathways and DNA damage response (DDR) pathways (6-8). In such evolving context, the identification of reliable biomarkers is a crucial challenge for laying the foundation of personalized medicine in SCLC (8).

High tumor mutational burden (TMB) induced by tobacco exposure is generally observed in SCLC and it certainly represents a strong rationale for immunotherapy. In fact, in the phase I/II CheckMate-032 study, nivolumab monotherapy and the combination of nivolumab plus ipilimumab showed promising antitumor activity and durable responses in pretreated patients, with median OS of 4.1 and 7.9 months respectively (9). Interestingly, an exploratory analysis reported an impressive median OS of 22 months for patients with high TMB treated with nivolumab plus ipilimumab (10). In the randomized, phase I/III IMpower133 study, the addition of atezolizumab to standard first-line carboplatin and etoposide extended median PFS from 4.3 to 5.2 months and median OS from 10.3 to 12.3 months as compared with carboplatin and etoposide plus placebo, thus becoming a standard option for first-line treatment (11). In this study, however, no association between blood-based TMB levels and the benefit of atezolizumab was found. Several ongoing clinical trials are currently investigating the role of immune checkpoint inhibitors and combinations in first-line, second-line and as maintenance therapy and possibly they also will further explore TMB as a biomarker for patients receiving immunotherapy.

Among developmental regulatory pathways, Notch is the most promising candidate as therapeutic target (6). Particularly, delta-like protein 3 (DLL3), an inhibitory 
Notch ligand involved in the embryonal development of central nervous system, is highly upregulated in SCLC but it is not expressed in normal adult tissues, thus representing an ideal target and also a potential biomarker. A phase I trial on patients with recurrent metastatic SCLC treated with rovalpituzumab tesirine (Rova-T), an antibodydrug conjugate that specifically targets DLL3, reported a response rate of $17 \%$ and a median OS of 4.6 months in the overall population (12). Among the 29 patients with high expression of DLL3, defined as detectable protein expression by immunohistochemistry in $\geq 50 \%$ of tumor cells, the activity of Rova-T was remarkable with a response rate of $35 \%$ and a median OS of 5.8 months. Notably, only patients with high DLL3 expression achieved an objective response, and this observation supports the role of DLL3 expression as a predictive biomarker for Rova-T. Based on these results, in the phase II TRINITY study (13) Rova-T was further investigated as third-line or later-line treatment for patients with DLL3-positive SCLC (defined as SCLC with DLL3 expression in $\geq 25 \%$ of tumor cells). In this study on heavily pretreated patients, investigator-assessed response rate was $18 \%$ and median OS was 5.6 months, with better outcomes reported for patients treated in thirdline and with higher levels of DDL3 expression ( $\geq 75 \%$ of tumor cells). Rova-T is currently under evaluation in two phase III trials as second-line treatment and as maintenance therapy respectively, and it is also being investigated in combination with chemotherapy and with checkpoint inhibitors in phase I trials.

In the past few years, growing evidence have also suggested the therapeutic opportunity of targeting DDR in SCLC $(7,8)$. Most cases of SCLC harbor inactivating mutations in TP53 and $R B$ genes as well as amplification of the oncogenic transcription factors $M Y C$ and $S O X-1$, thus resulting in frenetic cell proliferation and relevant DNA replication stress (7). In this context of genomic instability, survival of cancer cells is highly dependent on functional DDR and cell cycle checkpoints. Particularly the polyADP-ribose polymerase (PARP) enzymes play a key role in DDR primarily through the break excision repair (BER) pathway and they are more frequently upregulated in SCLC as compared to normal lung or NSCLC (8). Based on this background, PARP inhibition in SCLC could directly lead to tumor cell death or potentiate the cytotoxic effect of other anticancer drugs $(7,8)$. Therefore, PARP inhibitors have been actively investigating in SCLC, both as single agents and in combination with other anticancer drugs (Table 1).

\section{Temozolomide plus veliparib for relapsed SCLC}

On August 2018, Fournal of Clinical Oncology published the results of a randomized, double-blind, phase II study of temozolomide in combination with veliparib or placebo in patients with relapsed SCLC, by Pietanza and colleagues (14).

Temozolomide is an oral alkylating agent that produces $\mathrm{O}^{6}$-alkyl-guanine lesions on DNA, which are removed by the DNA-repair enzyme $\mathrm{O}^{6}$-methylguanine DNA methyltransferase (MGMT). Left unrepaired, temozolomide-induced DNA damage leads to DNA double-strand breaks, with subsequent inhibition of DNA replication and trigger of cell apoptosis. In a previous study, single-agent temozolomide had demonstrated activity in patients affected by relapsed SCLC with a response rate of $20 \%$ in the overall population, higher for patents with methylated $M G M T$ compared to those with unmethylated MGMT (38\% vs. 7\%, $\mathrm{P}=0.008$ ). However, the benefit obtained by single-agent temozolomide was short, with a median duration of response of 3.5 months and a median OS of 6 months, possible due to development of early resistance (15). It is well known that PARP-dependent BER pathway is involved in resistance to temozolomide, and this provided the rationale for investigating the combination of the PARP inhibitor veliparib plus temozolomide, with the aim to overcome resistance.

In this study, 104 patients with recurrent SCLC were randomized 1:1 to receive veliparib or placebo $40 \mathrm{mg}$ twice daily on days $1-7$, plus temozolomide $200 \mathrm{mg} / \mathrm{m}^{2} /$ day on days $1-5$ of a 28 -day cycle (14). After the first 24 patients were enrolled in the trial, grade $3 / 4$ hematologic toxicity was reported in 14 patients (included a case of grade 4 febrile neutropenia leading to sepsis and death in the temozolomide/veliparib arm), therefore protocol was amended to reduce temozolomide at $150 \mathrm{mg} / \mathrm{m}^{2} /$ day in order to avoid myelosuppression and treatment delays. Primary endpoint of the study was improvement of PFS at 4 months in patients receiving temozolomide/veliparib compared with temozolomide/placebo. Secondary objectives were response rate and OS, and exploratory objectives included PARP-1 and Schlafen-11 (SLFN11) immunohistochemical expression, MGMT promoter methylation, circulating tumor cells (CTCs) quantification and mutation analysis in DDR genes.

Formally, this was a negative study since 4-month PFS was not significantly improved in the temozolomide/ veliparib arm compared with temozolomide/placebo arm (36\% vs. $27 \%, \mathrm{P}=0.19)$ (14). Median OS also was not 
Table 1 Ongoing clinical trials with PARP inhibitors in SCLC

\begin{tabular}{|c|c|c|c|c|}
\hline Study identifier & Study phase & Setting & $\begin{array}{c}\text { Estimated } \\
\text { enrollment (pts) }\end{array}$ & Treatment \\
\hline NCT02289690 & $\mathrm{I} / \mathrm{II}$, randomized & $1^{\text {st }}$-line ES-SCLC & 221 & $\begin{array}{l}\text { Carboplatin + etoposide + veliparib vs. } \\
\text { carboplatin + etoposide }\end{array}$ \\
\hline NCT01642251 & $\mathrm{I} / \mathrm{II}$, randomized & $\begin{array}{l}1^{\text {st }} \text {-line ES-SCLC and } \\
\text { metastatic neuroendocrine NSCLC }\end{array}$ & 157 & $\begin{array}{l}\text { Cisplatin + etoposide + veliparib vs. } \\
\text { cisplatin + etoposide + placebo }\end{array}$ \\
\hline NCT02899728 & II, randomized & $\begin{array}{l}\text { Maintenance after } 1^{\text {st }} \text {-line } \\
\text { chemotherapy for ES-SCLC }\end{array}$ & 132 & Cediranib + olaparib \\
\hline NCT02769962 & $\mathrm{I} / \mathrm{II}$ & Recurrent ES-SCLC & 138 & CRLX101 + olaparib \\
\hline NCT03227016 & $\mathrm{I} / \mathrm{II}$ & Recurrent ES-SCLC & 130 & Topotecan + veliparib \\
\hline NCT03428607 & II & Recurrent ES-SCLC & 45 & AZD6738 + olaparib \\
\hline NCT02511795 & $\mathrm{lb}$ & Recurrent ES-SCLC & 135 & AZD1775 + olaparib \\
\hline NCT02937818 & II & Recurrent ES-SCLC & 91 & $\begin{array}{c}\text { Durvalumab + tremelimumab, AZD1775 + } \\
\text { carboplatin, AZD6738 + olaparib }\end{array}$ \\
\hline NCT03009682 & II & $\begin{array}{l}\text { Recurrent ES-SCLC with HR } \\
\text { pathway mutations }\end{array}$ & 28 & Olaparib \\
\hline NCT02734004 & $\mathrm{I} / \mathrm{II}$ & Solid tumors (including SCLC) & 288 & Olaparib + MEDI4736 +/- bevacizumab \\
\hline NCT02498613 & II & Solid tumors (including SCLC) & 126 & Cediranib + olaparib \\
\hline
\end{tabular}

PARP, poly-ADP-ribose polymerase; ES, extensive-stage; SCLC, small-cell lung cancer; NSCLC, non-small cell lung cancer; HR, homologous recombination.

significantly different between the two arms (8.2 versus 7.0 months; $\mathrm{P}=0.50)$. Even if the temozolomide/veliparib combination achieved higher response rate compared with temozolomide/placebo ( $39 \%$ vs. $14 \%, \mathrm{P}=0.016$ ), this came at the cost of higher hematologic toxicity, particularly grade $3 / 4$ thrombocytopenia (50\% vs. $9 \%$ ), grade $3 / 4$ neutropenia ( $31 \%$ vs. $7 \%$ ), and febrile neutropenia (4\% vs. $0 \%$ ). As the authors themselves mentioned in the discussion section of the paper, hematologic toxicities were often observed early in the temozolomide/veliparib arm thus leading to treatment delays, and this may potentially explain the lack of benefit with the combination. Another possible explanation for the negative results lies in the dose levels chosen for veliparib and temozolomide. In this trial, in fact, veliparib was given at low dose on the basis of the results of a phase II study in breast cancer (16) and temozolomide was given at the full recommended dose in SCLC, whereas more recent data suggested that, on the contrary, the optimal synergy may be achieved with a near-maximal dose of PARP inhibitors plus a submaximal dose of temozolomide.

Although this was a negative study, interesting data came from the exploratory biomarker analysis (14). Briefly, PARP-1 expression did not correlate with outcome. No definitive conclusion could be drawn on the role of MGMT promoter methylation as well as on the role of mutations in DDR genes, due to the low number of tissue samples analyzed (32 samples and 22 samples, respectively, for MGMT methylation analysis and targeted sequencing of DDR genes). A prognostic rather than predictive role was suggested for CTCs, given that elevated levels ( $\geq 5$ CTCs) both at baseline and after first cycle were associated with worse OS at the univariable analysis.

The most relevant results of the biomarker analysis involved SLFN11 (14). SLFN11 is a DNA/RNA helicase that is actively recruited to the sites of DNA damage and regulates replication stress (8). Preclinical data indicated 
SLFN11 as a candidate marker of sensitivity to DNAdamaging chemotherapy and PARP inhibitors. Particularly, among 63 SCLC and 3 NSCLC cell lines, high SLFN11 expression was found to be associated with sensitivity to PARP inhibitors (17). This finding was further confirmed in vivo on SCLC patient-derived xenograft models (18). Based on these findings, with a protocol amendment the immunohistochemistry assessment of SFLN11 was included in the exploratory objectives of the study (14). Among 48 tumor samples evaluated for SFLN11 expression 23 were SFLN11-positive (H-score $\geq 1)$ and 25 were SFLN11negative $(\mathrm{H}$-score $<1)$. In terms of response rate, there was no significant difference on the basis of SLFN11 expression, in either study arms. However, patients with SFLN-positive tumors treated with temozolomide/veliparib had prolonged PFS (5.7 vs. 3.6 months, $\mathrm{P}=0.009$ ) and $\mathrm{OS}$ (12.2 vs. 7.5 months, $\mathrm{P}=0.014$ ) compared to those with SLFN11-negative tumors, whereas no difference in terms of PFS or OS were observed in the temozolomide/placebo arm on the basis of SLFN11 expression. The reason for a PFS and OS benefit without an increase in objective response for patients with SLFN11-positve tumors treated with temozolomide/veliparib is not completely clear, but a trend towards deeper responses and also possibly a longer duration of response may represent an explanation.

Despite the limitations of the assessment of SLFN11 in this study, including its exploratory nature and the limited sample size (less than $50 \%$ of patients enrolled in the study were assessed for SLFN11), the median OS of approximatively 12 months reported for patients with SLFN11-positive disease is impressive (14), and the consistency between the preclinical background and clinical trial results increase the plausibility that SLFN11 may represent a predictive biomarker for PARP inhibitors in SCLC.

\section{Conclusions}

Immune checkpoint inhibitors and antibody-drug conjugates have recently entered the treatment landscape of SCLC, and other agents are on horizon. In particular, PARP inhibitors are being tested in a number of clinical trials recruiting more than one-thousand patients. Possibly, a biomarker-driven selection of patients could improve clinical trial results. In this perspective, the data reported by Pietanza and colleagues are extremely relevant. For the first time in a clinical trial, in fact, it was observed that high expression if SLFN11 could serve as predictive biomarker of effectiveness of PARP inhibitors in SCLC. This finding, however, derived from an exploratory analysis on a limited sample size and warrants further investigation. If the predictive role of SLFN11 will be confirmed in welldesigned biomarker validation studies, it could represent an important step towards personalized medicine in SCLC.

\section{Acknowledgements}

None.

\section{Footnote}

Conflicts of Interest: The authors have no conflicts of interest to declare.

\section{References}

1. Byers LA, Rudin CM. Small cell lung cancer: where do we go from here? Cancer 2015;121:664-72.

2. Niell HB, Herndon JE 2nd, Miller AA, et al. Randomized phase III intergroup trial of etoposide and cisplatin with or without paclitaxel and granulocyte colony-stimulating factor in patients with extensive-stage small-cell lung cancer: Cancer and Leukemia Group B Trial 9732. J Clin Oncol 2005;23:3752-9.

3. Rossi A, Di Maio M, Chiodini P, et al. Carboplatin- or cisplatin-based chemotherapy in first-line treatment of small-cell lung cancer: the COCIS meta-analysis of individual patient data. J Clin Oncol 2012;30:1692-8.

4. Hanna N, Bunn PA Jr, Langer C, et al. Randomized phase III trial comparing irinotecan/cisplatin with etoposide/ cisplatin in patients with previously untreated extensivestage disease small-cell lung cancer. J Clin Oncol 2006;24:2038-43.

5. Horita N, Yamamoto M, Sato T, et al. Topotecan for Relapsed Small-cell Lung Cancer: Systematic Review and Meta-Analysis of 1347 Patients. Sci Rep 2015;5:15437.

6. Sabari JK, Lok BH, Laird JH, et al. Unravelling the biology of SCLC: implications for therapy. Nat Rev Clin Oncol 2017;14:549-61.

7. Foy V, Schenk MW, Baker K, et al. Targeting DNA damage in SCLC. Lung Cancer 2017;114:12-22.

8. Sen T, Gay CM, Byers LA. Targeting DNA damage repair in small cell lung cancer and the biomarker landscape. Transl Lung Cancer Res 2018;7:50-68.

9. Antonia SJ, López-Martin JA, Bendell J, et al. Nivolumab alone and nivolumab plus ipilimumab in recurrent small- 
cell lung cancer (CheckMate 032): a multicentre, openlabel, phase 1/2 trial. Lancet Oncol 2016;17:883-95.

10. Hellmann MD, Callahan MK, Awad MM, et al. Tumor Mutational Burden and Efficacy of Nivolumab Monotherapy and in Combination with Ipilimumab in Small-Cell Lung Cancer. Cancer Cell 2018;33:853-61.e4.

11. Horn L, Mansfield AS, Szczęsna A, et al. First-Line Atezolizumab plus Chemotherapy in Extensive-Stage Small-Cell Lung Cancer. N Engl J Med 2018. [Epub ahead of print].

12. Rudin CM, Pietanza MC, Bauer TM, et al. Rovalpituzumab tesirine, a DLL3-targeted antibody-drug conjugate, in recurrent small-cell lung cancer: a first-inhuman, first-in-class, open-label, phase 1 study. Lancet Oncol 2017; 18:42-51.

13. Carbone DP, Morgensztern D, Le Moulec S, et al. Efficacy and safety of rovalpituzumab tesirine in patients With DLL3-expressing, $\geq 3$ rd line small cell lung cancer: Results from the phase 2 TRINITY study. J Clin Oncol 2018;36:8507.

14. Pietanza MC, Waqar SN, Krug LM, et al. Randomized,

Cite this article as: Inno A, Stagno A, Gori S. Schlafen-11 (SLFN11): a step forward towards personalized medicine in small-cell lung cancer? Transl Lung Cancer Res 2018;7(Suppl 4):S341-S345. doi: 10.21037/tlcr.2018.11.06
Double-Blind, Phase II Study of Temozolomide in Combination With Either Veliparib or Placebo in Patients With Relapsed-Sensitive or Refractory Small-Cell Lung Cancer. J Clin Oncol 2018;36:2386-94.

15. Pietanza MC, Kadota K, Huberman K, et al. Phase II trial of temozolomide in patients with relapsed sensitive or refractory small cell lung cancer, with assessment of methylguanine-DNA methyltransferase as a potential biomarker. Clin Cancer Res 2012;18:1138-45.

16. Isakoff SJ, Overmoyer B, Tung NM, et al: A phase II trial of the PARP inhibitor veliparib (ABT888) and temozolomide for metastatic breast cancer. J Clin Oncol 2010;28:1019.

17. Polley E, Kunkel M, Evans D, et al. Small Cell Lung Cancer Screen of Oncology Drugs, Investigational Agents, and Gene and microRNA Expression. J Natl Cancer Inst 2016;108(10).

18. Lok BH, Gardner EE, Schneeberger VE, et al. PARP Inhibitor Activity Correlates with SLFN11 Expression and Demonstrates Synergy with Temozolomide in Small Cell Lung Cancer. Clin Cancer Res 2017;23:523-35. 